On the validity of the five-dimensional Birkhoff theorem: the tale of an exceptional case

This content has been downloaded from IOPscience. Please scroll down to see the full text. 2008 Class. Quantum Grav. 25165016

(http://iopscience.iop.org/0264-9381/25/16/165016)

View the table of contents for this issue, or go to the journal homepage for more

Download details:

IP Address: 134.153.184.170

This content was downloaded on 16/07/2014 at 22:00

Please note that terms and conditions apply. 


\title{
On the validity of the five-dimensional Birkhoff theorem: the tale of an exceptional case
}

\author{
Zoltán Keresztes $^{1}$ and László Á Gergely ${ }^{1,2}$ \\ ${ }^{1}$ Departments of Theoretical and Experimental Physics, University of Szeged, Dóm tér 9, \\ Szeged 6720, Hungary \\ ${ }^{2}$ Department of Applied Science, London South Bank University, 103 Borough Road, \\ London SE1 OAA, UK \\ E-mail: zkeresztes@titan.physx.u-szeged.hu and gergely@physx.u-szeged.hu
}

Received 21 December 2007, in final form 2 June 2008

Published 5 August 2008

Online at stacks.iop.org/CQG/25/165016

\begin{abstract}
The five-dimensional (5d) Birkhoff theorem gives the class of $5 \mathrm{~d}$ vacuum spacetimes containing spatial hypersurfaces with cosmological symmetries. This theorem is violated by the $5 \mathrm{~d}$ vacuum Gergely-Maartens (GM) spacetime, which is not a representant of the above class, but contains the static Einstein brane as embedded hypersurface. We prove that the $5 \mathrm{~d}$ Birkhoff theorem is still satisfied in a weaker sense: the GM spacetime is related to the degenerated horizon metric of certain black hole spacetimes of the allowed class. This result resembles the connection between the Bertotti-Robinson spacetime and the horizon region of the extremal Reissner-Nordstrom spacetime in general relativity.
\end{abstract}

PACS numbers: $04.50 . \mathrm{Gh}, 04.50 .-\mathrm{h}, 11.25 . \mathrm{Uv}, 04.20 . \mathrm{Jb}$

\section{Introduction}

In the most simple brane-world models the brane to which the standard model fields are confined is embedded into a five-dimensional (5d) spacetime, in which only gravity acts. The basic dynamical equation on the brane is the effective Einstein equation [1], supplemented by the Codazzi and twice-contracted Gauss equations [2]. For a general overview of brane-worlds see [3].

Such models admit black hole solutions with tidal charge on the brane [4]. The tidal charge represents the effect of the Kaluza-Klein modes of gravity from the extra dimension, however the $5 \mathrm{~d}$ spacetime in which such a brane is embedded, is still unknown. Stars on a brane [5-7] and gravitational collapse under spherical symmetry [8-13] were also studied in brane-worlds, yielding to striking features like the production of radiation in a spherically 
symmetric collapse [10] and the emergence of unconventional forms of stellar matter leading to dark energy production below the horizon [13].

Cosmological brane-world models were also studied, like a Gödel brane [14], branes with Swiss-cheese type inhomogeneities $[15,16]$, but the most important, brane-world models with Friedmann branes [2, 17], among them the static Einstein brane [18] $]^{3}$.

The Einstein static universe containing a perfect fluid is widely known to be unstable against spatially homogeneous and isotropic perturbations [19]. However recent systematic analysis using covariant techniques [20] has shown that it is neutrally stable against small inhomogeneous vector and tensor perturbations and neutrally stable against adiabatic scalar density inhomogeneities for the velocity of sound obeying $c_{s}^{2}>1 / 5$. The stability of Einstein universes was also considered in alternative gravitational theories, like the Einstein brane in the DGP model [21], in $f(R)$ gravity [22] and in loop quantum cosmology [23].

The most general static vacuum $5 \mathrm{~d}$ spacetime with cosmological constant $\widetilde{\widetilde{\Lambda}}=3 \varepsilon \Gamma^{2} / \widetilde{\kappa}^{2}$ ( $\varepsilon$ carries the sign of $\widetilde{\Lambda}$ and $\widetilde{\kappa}^{2}$ is the gravitational constant in $5 \mathrm{~d}$ ), which contains a Friedmann brane and has the symmetries of the brane in each point is [24] (see also [25])

$\mathrm{d} \widetilde{s}^{2}=-f(r ; k, \varepsilon) \mathrm{d} t^{2}+\frac{\mathrm{d} r^{2}}{f(r ; k, \varepsilon)}+r^{2}\left[\mathrm{~d} \chi^{2}+\mathcal{H}^{2}(\chi ; k)\left(\mathrm{d} \theta^{2}+\sin ^{2} \theta \mathrm{d} \varphi^{2}\right)\right]$,

with the metric functions

$$
f(r ; k, \varepsilon)=k-\frac{2 m}{r^{2}}-\frac{\varepsilon \Gamma^{2}}{2} r^{2}
$$

and

$$
\mathcal{H}(\chi ; k)= \begin{cases}\sin \chi, & k=1 \\ \chi, & k=0 \\ \sinh \chi, & k=-1,\end{cases}
$$

where both $\varepsilon$ and $k$ take any of the values $(0, \pm 1)$. This result is frequently referred as the $5 \mathrm{~d}$ Birkhoff theorem.

However an interesting exceptional case has been found in [18], representing a family of vacuum solutions of the $5 \mathrm{~d}$ Einstein equations with cosmological constant $\widetilde{\Lambda}$ which contain an Einstein brane. This $5 \mathrm{~d}$ spacetime is given for $y>0$ as

$$
\Gamma^{2} \mathrm{~d} \widetilde{s}^{2}=-F^{2}(y ; \varepsilon) \mathrm{d} \tau^{2}+\mathrm{d} y^{2}+\mathrm{d} \chi^{2}+\mathcal{H}^{2}(\chi ; \varepsilon)\left(\mathrm{d} \theta^{2}+\sin ^{2} \theta \mathrm{d} \varphi^{2}\right)
$$

The boundary at $y=0$ is the static Einstein brane [18], therefore a particular case of the Friedmann branes for which the $5 \mathrm{~d}$ Birkhoff theorem refers. The metric functions are

$$
F(y ; \varepsilon)= \begin{cases}A \cos (\sqrt{2} y)+B \sin (\sqrt{2} y), & \varepsilon=1 \\ A+\sqrt{2} B y & \varepsilon=0 \\ A \cosh (\sqrt{2} y)+B \sinh (\sqrt{2} y), & \varepsilon=-1,\end{cases}
$$

and $\mathcal{H}(\chi ; \varepsilon)$ defined as (3), with $\varepsilon$ in place of $k$. A homogeneous counterpart of the GergelyMaartens (GM) metric (5) was also found [26]. The GM metric is well defined on the brane for any $A \neq 0$. According to the Lanczos equation a vanishing $B$ would be incompatible with brane matter [18]. As any of the constants $A$ or $B$ can be absorbed into the coordinate $\tau$, the GM metric represents a one-parameter family of solutions, which is not a sub-case of the metrics (1).

The higher dimensional Birkhoff theorem was formulated in an alternative way in [27], by enouncing the set of conditions under which the higher dimensional spacetime is static.

3 The correspondence to the notations of [18] is: $\left(\alpha, \beta^{2}, \mu, t, K_{\mathbf{i}}\right) \rightarrow\left(\operatorname{sgn}\left(D^{2}\right),\left|D^{2}\right|, 2 m, \tau, K_{\mathbf{i}}^{\mathrm{GM}}\right)$. In the round bracket of the expression of $K_{\mathbf{8}}^{\mathrm{GM}}$ given in (A1) the $\delta_{\alpha}^{0}$ term should have an additional factor of $A / B$ and the sign of the first term in the commutator $\left[K_{8}, K_{9}\right]$ in (A6) is positive. 
The GM metric does not obey these conditions either as its metric coefficient $g_{\chi \chi}$ is a constant. Therefore it stays outside the validity of theorem 1 of [27] .

As remarked in section 4 of [18], the proof presented in [24] leading to metric (1) cannot be applied when the metric function $B$ of [24] (which is different from the parameter $B$ of metric (4)) is a constant. Then it is not possible to introduce $r=B^{1 / 3}$ as a new radial coordinate in order to obtain the class of metrics (1). This suggests however that the GM metric may be related to a tiny layer $(r, r+\mathrm{d} r)$ of the spacetimes (1). It is the purpose of the present paper to prove this conjecture and re-establish the validity of the $5 \mathrm{~d}$ Birkhoff theorem in a weaker sense.

In section 2, we will enlist arguments in favour of the claim that the GM spacetime is related to the horizon regions of certain 5d black hole metrics (1). We also write up an approximate form of the black hole metrics (1), valid in the vicinity of the degenerated horizons of (1). We present the horizons of the various metrics in class (1) in appendix A. Section 3 contains the technical derivation of the coordinate transformation bringing the black hole horizon metric into the GM spacetime, done explicitly for various subcases of the parameters of the GM metric. Section 4 contains discussions on the equivalence of the GM metric and horizon metric, based on the analysis of the Killing algebras, presented in appendix B. Section 5 is the concluding remarks. In appendix $\mathrm{C}$ we present a related result from general relativity: the Bertotti-Robinson solution [28, 29] describing gravity in the presence of a covariantly constant electromagnetic field corresponds to the horizon region of the extremal Reissner-Nordström black hole [30]. We present this both for didactical reasons, as this derivation is not well known, and as a simpler analogy for the method we follow in section 3 .

\section{Black hole horizons in a 5d spacetime with Friedmann brane boundary}

It is immediate to see from the $(\chi, \theta, \varphi)$ sector that any relation between the spacetimes (1) and (4) may exist only for $k=\varepsilon$. In what follows, we will discuss only such metrics from the class $(1)^{4}$. As noted in [18], the curvature scalar of the GM solution with $\varepsilon=-1$ agrees with the curvature scalar of the $5 \mathrm{~d}$ black hole metric (1) with $k=\varepsilon=-1$, only when evaluated at the horizon (when $m=-1 / 4 \Gamma^{2}$ ). This is a serious indication that the GM solution is related to the event horizon of certain 5 d black hole metrics.

No such relation exists in the non-cosmological case $(\varepsilon=0)$. Then the scalars $\widetilde{R}_{a b c d} \widetilde{R}^{a b c d}$ and $\widetilde{C}_{a b c d} \widetilde{C}^{a b c d}$ vanish in the GM spacetime, however in the spacetime (1) they are $\widetilde{R}_{a b c d} \widetilde{R}^{a b c d}=\widetilde{C}_{a b c d} \widetilde{C}^{a b c d}=288 \mathrm{~m}^{2} / r^{8}$. This can vanish for any finite value of $r$ only if $m=0$, but then the metric (1) becomes ill-defined.

In order to establish more exactly the connection between the GM spacetime and the horizon regions of the Schwarzschild-(anti) de Sitter metrics (1) we enlist the loci of the horizons (given by $f=0$ ) for various $\varepsilon$ in tables A1-A3 of appendix A. We note that there is no horizon in the case $\varepsilon=0$, so it is not surprising that in this case no connection can be established with the family of GM metrics.

There are two horizons only in the cases $\varepsilon=k=1, m>0$ or $\varepsilon=k=-1, m<0$. These horizons merge into one (degenerated) horizon at $\Gamma r=1$ for $\varepsilon m=1 / 4 \Gamma^{2}$. The latter is exactly the condition, under which for $\varepsilon=-1$ the curvature scalar of the $5 \mathrm{~d}$ black hole metric was shown to agree with the curvature scalar of the GM metric. Therefore we expect to find a correspondence between the GM metric and the degenerated horizon regions of the 5 d black hole metrics.

4 As we show in appendix A, only a subset of these metrics have horizons. In the cases $\mathrm{km}>0$ the metric (1) is also known as $5 \mathrm{~d}$ topological black hole $(\mathrm{TBH})$ for $\varepsilon=0$ or (anti) de Sitter TBH for $\varepsilon=1(\varepsilon=-1)$, see [31]. 
For this we introduce the new coordinate $\rho=\Gamma r-1$, which is small close to the degenerated horizon, positive above the horizon and $\rho \in(-1,0)$ below the horizon. For small $\rho$ the metric function $f$ has the approximate expression $f=-2 \varepsilon \rho^{2}$ and by rescaling the time coordinate as $t \rightarrow 4 \Gamma^{2} t$ we obtain the 'horizon metric'

$$
\Gamma^{2} \mathrm{~d} \widetilde{s}^{2}=\frac{\varepsilon}{2}\left(\rho^{2} \mathrm{~d} t^{2}-\frac{\mathrm{d} \rho^{2}}{\rho^{2}}\right)+\mathrm{d} \chi^{2}+\mathcal{H}^{2}(\chi ; \varepsilon)\left(\mathrm{d} \theta^{2}+\sin ^{2} \theta \mathrm{d} \varphi^{2}\right),
$$

describing for $\varepsilon=k=1$ ( or $\varepsilon=k=-1$ ) the vicinity of the horizon of the Schwarzschild—de Sitter (or Schwarzschild-anti de Sitter-like with $k=-1$ ) spacetime. The time coordinate for $\varepsilon=1$ is $\rho$ and for $\varepsilon=-1$ is $t$. We give the Killing vectors and Killing algebra of the horizon metric in appendix B. As the horizon metric solves the five-dimensional Einstein equations in the presence of a cosmological constant $\widetilde{\Lambda}=3 \varepsilon \Gamma^{2} / \widetilde{\kappa}^{2}$, it can be extended towards non-small values of $\rho$ either.

\section{The relation between the GM and the horizon metrics}

In this section we prove that for $\varepsilon= \pm 1$ the GM spacetime is related to the degenerated horizon region (6) of the $5 \mathrm{~d}$ black hole (1) with the same $\varepsilon$ and $k=\varepsilon$. In order to compare the degenerated horizon region (6) of the $5 \mathrm{~d}$ black hole metric (1) with the GM metric, we absorb its parameter $A$ into $\tau$ and denote $B / A$ with $B$. Then we rewrite the metric function $F(y ; \varepsilon= \pm 1)$ as

$$
\begin{aligned}
& F(y ; \varepsilon)=\cos z+\beta \sin z, \quad \varepsilon= \pm 1, \\
& z(y ; \varepsilon)=\sqrt{2} \mathrm{i}^{(1-\varepsilon) / 2} y \\
& \beta(B ; \varepsilon)=(-\mathrm{i})^{(1-\varepsilon) / 2} B .
\end{aligned}
$$

Next we try to identify a suitable coordinate transformation $(t, \rho) \rightarrow(\tau, y)$ of the horizon metric. In order to enforce the correspondence with the GM spacetime, the original coordinates $t(\tau, y), \rho(\tau, y)$ have to obey the following differential equations:

$$
\begin{aligned}
& \rho^{2}\left(\frac{\partial t}{\partial \tau}\right)^{2}-\frac{1}{\rho^{2}}\left(\frac{\partial \rho}{\partial \tau}\right)^{2}=-2 \varepsilon F^{2}(y ; \varepsilon), \\
& \rho^{2}\left(\frac{\partial t}{\partial \tau}\right)\left(\frac{\partial t}{\partial y}\right)-\frac{1}{\rho^{2}}\left(\frac{\partial \rho}{\partial \tau}\right)\left(\frac{\partial \rho}{\partial y}\right)=0, \\
& \rho^{2}\left(\frac{\partial t}{\partial y}\right)^{2}-\frac{1}{\rho^{2}}\left(\frac{\partial \rho}{\partial y}\right)^{2}=2 \varepsilon .
\end{aligned}
$$

For separable solutions $t=t_{0}(\tau) t_{1}(y)$ and $\rho=\rho_{0}(\tau) \rho_{1}(y)$ the system (8) simplifies to

$$
\begin{aligned}
& \left(\rho_{0} \dot{t}_{0}\right)^{2}\left(\rho_{1} t_{1}\right)^{2}-\left(\frac{\dot{\rho}_{0}}{\rho_{0}}\right)^{2}=-2 \varepsilon F^{2}(y ; \varepsilon), \\
& \left(\rho_{0}^{2} t_{0} \dot{t}_{0}\right)\left(\rho_{1}^{2} t_{1} t_{1}^{\prime}\right)-\left(\frac{\dot{\rho}_{0}}{\rho_{0}}\right)\left(\frac{\rho_{1}^{\prime}}{\rho_{1}}\right)=0, \\
& \left(\rho_{0} t_{0}\right)^{2}\left(\rho_{1} t_{1}^{\prime}\right)^{2}-\left(\frac{\rho_{1}^{\prime}}{\rho_{1}}\right)^{2}=2 \varepsilon
\end{aligned}
$$

where a dot (a prime) denotes the derivative with respect to $\tau(y)$. In the last equation only $\rho_{0}^{2} t_{0}^{2}$ depends on $\tau$, therefore either (a) $t_{1}^{\prime}=0$, thus $t=t(\tau)$ or (b) $\rho_{0} t_{0}=$ const. We consider these cases separately: 
(a) When $t=t(\tau)$ from $(9 c)$

$$
\rho_{1}=C_{1} \exp ( \pm \sqrt{-2 \varepsilon} y)
$$

$\left(C_{1}\right.$ a constant). Equation $(9 b)$ gives $\rho_{0}(\tau)=C_{0}$ (a constant). Substituting $\rho$ into $(9 a)$ finally we get

$$
C_{0} C_{1} \exp ( \pm \sqrt{-2 \varepsilon} y)\left(\frac{\mathrm{d} t}{\mathrm{~d} \tau}\right)=\sqrt{-2 \varepsilon} F(y ; \varepsilon)
$$

Thus $\mathrm{d} t / \mathrm{d} \tau$ must be another constant, say $C_{2}$ and

$$
F(y ; \varepsilon)=\frac{C_{0} C_{1} C_{2}}{\sqrt{-2 \varepsilon}} \exp ( \pm \sqrt{-2 \varepsilon} y)
$$

For the particular values of the constants $C_{0} C_{1} C_{2}=\sqrt{2}$ and for $\varepsilon=-1$ this becomes the metric function (7) for $B= \pm 1$

$$
F(y ;-1)=\exp ( \pm \sqrt{2} y)=\cosh \sqrt{2} y \pm \sinh \sqrt{2} y .
$$

If we additionally choose $C_{2}=1$, then

$$
t=\tau, \quad \rho=\sqrt{2} \exp ( \pm \sqrt{2} y)
$$

is a coordinate transformation from the degenerated horizon metric (6) with $\varepsilon=-1$ into the GM spacetime with $\varepsilon=-1$ and $B= \pm 1$.

(b) For $t_{1}^{\prime} \neq 0$ and $\rho_{0} t_{0}=C_{3} \neq 0$ (a constant) equation ( $9 a$ ) becomes

$$
\frac{\dot{\rho}_{0}^{2}}{\rho_{0}^{2}}=\frac{-2 \varepsilon F^{2}(y ; \varepsilon)}{\left(\rho_{1}^{2} t_{1}^{2} C_{3}^{2}-1\right)} .
$$

This gives $\dot{\rho}_{0} / \rho_{0}=-D$ (a constant), thus

$$
\rho_{0}=C_{4} \exp (-D \tau), \quad t_{0}=\frac{C_{3}}{C_{4}} \exp (D \tau),
$$

where $C_{4}$ is the integration constant. Equation (15) also implies

$$
t_{1}^{2}=\frac{D^{2}-2 \varepsilon F^{2}(y ; \varepsilon)}{C_{3}^{2} D^{2} \rho_{1}^{2}},
$$

and from $\rho_{0} t_{0}=C_{3}$ and equation $(9 b)$

$$
\rho_{1}^{2} t_{1} t_{1}^{\prime} C_{3}^{2}+\frac{\rho_{1}^{\prime}}{\rho_{1}}=0
$$

The last two equations imply

$$
\frac{\rho_{1}^{\prime}}{\rho_{1}}=\frac{F^{\prime}(y ; \varepsilon)}{F(y ; \varepsilon)},
$$

with solution

$$
\rho_{1}=G F(y ; \varepsilon)
$$

where $G$ is an integration constant. Finally equation $(9 c)$ constraints $F(y ; \varepsilon)$ as

$$
2 \varepsilon F^{\prime}(y ; \varepsilon)^{2}+4 F(y ; \varepsilon)^{2}-2 \varepsilon D^{2}=0 .
$$


The metric function (7) solves this equation for $D^{2}=2\left(B^{2}+\varepsilon\right)$. Thus for this value of $D$ the horizon metric transforms into the GM metric under the coordinate transformation

$\rho=\sqrt{2} \exp (-D \tau) F(y ; \varepsilon), \quad t=\frac{(-\mathrm{i})^{(1-\varepsilon) / 2}}{\sqrt{2}\left(B^{2}+\varepsilon\right)^{1 / 2}} \exp (D \tau) \frac{\tan z-\beta}{1+\beta \tan z}$.

(We have set $C_{4} G=\sqrt{2}$.)

We note that the result derived in (a) only partially emerges from the limit $D \rightarrow 0$ of the result derived in (b) specified for $\varepsilon=-1$ and $B= \pm 1$ (thus $D=0$ ) in the following sense. First, for $D=0$ the metric function (13) solves the differential equation (21). Second, the expression of $\rho$ from (22) reduces to the corresponding expression (14), however the transformation from $t$ to $\tau$ differs in a shift $1 / D \rightarrow \infty$.

\section{Discussion}

The transformation (22) admits the following three particular cases:

(b1) Case $\varepsilon=1$. Then the horizon coordinates $(t, \rho)$ are related by a real coordinate transformation to the GM coordinates $(\tau, y)$ :

$\rho=D \exp (-D \tau) \cos \left(\alpha_{1}+\sqrt{2} y\right), \quad t=\frac{1}{D} \exp (D \tau) \tan \left(\alpha_{1}+\sqrt{2} y\right)$,

where we have denoted $B=-\tan \alpha_{1}$. We also note that this transformation obeys $t^{2} \rho^{2}<1$, thus the GM spacetime only partially covers the horizon spacetime $(6)^{5}$.

Thus the transformation (23) links the $t^{2} \rho^{2}<1$ region of the $5 \mathrm{~d}$ black hole horizon metric with $\varepsilon=1$ to the GM metric with $\varepsilon=1$. In this region ${ }^{6}$ the horizon metric is static due to $K_{\mathbf{8}}$, as shown in appendix B.

(b2) For $\varepsilon=-1$ and $B^{2}>1$ (implying $\operatorname{sgn}\left(D^{2}\right)=1$ ) the coordinate transformation is

$\rho=D \exp (-D \tau) \sinh \left(\alpha_{2}+\sqrt{2} y\right), \quad t=\frac{1}{D} \exp (D \tau) \operatorname{coth}\left(\alpha_{2}+\sqrt{2} y\right)$,

where we have denoted $B=\operatorname{coth} \alpha_{2}$. The horizon coordinates obey $t^{2} \rho^{2}>1$. The GM spacetime in this case also covers only partially the horizon spacetime (6). In this part of the horizon spacetime $K_{\mathbf{8}}$ is time-like, as well as $K_{7,9}$.

(b3) For $\varepsilon=-1$ and $B^{2}<1$ (implying $\operatorname{sgn}\left(D^{2}\right)=-1$ ) the horizon coordinates $(\rho, t)$ are related to the GM coordinates by a complex transformation

$\rho=-\mathrm{i} D \exp (-D \tau) \cosh \left(\alpha_{3}+\sqrt{2} y\right), \quad t=\frac{1}{D} \exp (D \tau) \tanh \left(\alpha_{3}+\sqrt{2} y\right)$,

with $B=\tanh \alpha_{3}$. Note that in this case $D$ is purely imaginary, which implies $t^{2} \rho^{2}<0$. The coordinate transformation being complex, this case is the closest analogue of the general relativistic result that the Bertotti-Robinson metric is related to the horizon region of the extremal Reissner-Nordström metric (see the striking similarity with the structure of equations (C.5) in appendix C).

Remembering that the transformation (14) classified as (a) relates the degenerated horizon metric of the $5 \mathrm{~d}$ black hole (6) with $\varepsilon=-1$ to the GM spacetime with $\varepsilon=-1$ and $B= \pm 1$, we see that all possible cases of the GM metric with the cosmological constant $(\varepsilon= \pm 1$,

5 This is similar to another famous example from brane-worlds, which establishes the equivalence of the static branes written in Gauss normal coordinates and moving branes in 5d Schwarzschild—anti de Sitter spacetime [32].

6 The $5 \mathrm{~d}$ black hole metric (1) for $\varepsilon=k=1$ and $m>0$ is static only between the horizons (which degenerate for $m=1 / 4 \Gamma^{2}$ ). In other words the Killing vector $K_{7}$ is time-like between the horizons, space-like outside. Thus for degenerated horizons there is no time-like Killing vector. However the approximate degenerate horizon metric (6) acquires new symmetries, among which $K_{\mathbf{8}}$ is time-like for $t^{2} \rho^{2}<1$. 
$B$ arbitrary) are covered by our analysis. All GM metrics with $\varepsilon=-1$ are covered in (a), (b2), (b3), while the GM metrics with $\varepsilon=1$ in (b1).

In the cases with $\varepsilon=-1$ the static character of the $5 \mathrm{~d}$ black hole metric both above and below the degenerated horizons is assured by $K_{7}$, which also remains a time-like Killing vector for the horizon metric. In all these cases $K_{\mathbf{9}}$ is also time-like, while $K_{\mathbf{8}}$ is time-like for (b2), space-like for (b3), and its causal character depends on the actual value of the coordinates for (a), as for this transformation (14) $\rho^{2} t^{2}>0$.

As remarked earlier, for $\varepsilon=1$ (case (b1)) the coordinate transformation (23) relates the static GM metric to the static region of the horizon metric.

In each case the GM metric can be related to the horizon metric either by a real (cases (a), (b1), (b2)), or by a complex coordinate transformation (case (b3)). The latter case is similar to the general relativistic analogy between the Bertotti-Robinson spacetime and the degenerated horizon region of the extremal Reissner-Nordström black hole shown by a complex transformation (see appendix C). Both there and in our case (b3) this is understood in the following sense: although the emerging coordinates are complex, only their real subset is considered in the line element.

\section{Concluding remarks}

We have shown that the 5d GM spacetime, which contains the Einstein brane as boundary, although violates the $5 \mathrm{~d}$ Birkhoff theorem (being a $5 \mathrm{~d}$ vacuum spacetime different from (1) and with an embedded static Friedmann brane), obeys the theorem in the following weaker sense. For all cases of the GM spacetime parameters a specific 5d black hole metric can be found for which the GM metric is related to its degenerated horizon region either by a real or a complex coordinate transformation. We have proven this result by explicitly constructing the respective coordinate transformations.

For a positive $5 \mathrm{~d}$ cosmological constant $(\varepsilon=1)$, the GM metric represents the static region of the horizon metric which approximates the 5d Schwarzschild-de Sitter 5d black hole degenerate horizon region.

A negative $5 \mathrm{~d}$ cosmological constant $(\varepsilon=-1)$ is far more acceptable from a brane point of view as it gives a small cosmological constant $\Lambda$ on the brane through the relation

$$
2 \Lambda=\kappa^{2} \lambda+3 \varepsilon \Gamma^{2},
$$

(where $\lambda=6 \kappa^{2} / \widetilde{\kappa}^{4}$ is the brane tension, known to have a high value [33], and $\kappa^{2}$ is the brane gravitational constraint). For this case we have shown that the GM metric is related to the static horizon metric representing the region close to the degenerated horizon of a $5 \mathrm{~d}$ Schwarzschild-anti de Sitter-like black hole with curvature index $k=-1$.

The generic result established in this paper according to which the GM spacetime containing the Einstein brane is the degenerated horizon region of the Schwarzschild(anti) de Sitter 5d black hole, is in close analogy with the general relativistic result, that the Bertotti-Robinson spacetime generated by a covariantly constant electromagnetic field is the degenerated horizon region of the extremal Reissner-Nordström spacetime.

Our result re-establishes the validity of the Birkhoff theorem in 5d, although in a weaker sense.

\section{Acknowledgments}

This work was supported by OTKA grants nos 46939 and 69036. LÁG was further supported by the János Bolyai Grant of the Hungarian Academy of Sciences. 
Table A1. The location (given by the $r$ coordinate) of the horizons with the vanishing $5 \mathrm{~d}$ cosmological constant $(\varepsilon=0)$.

\begin{tabular}{llll}
\hline & $m<0$ & $m=0$ & $m>0$ \\
\hline$k=-1$ & $\sqrt{-2 m}$ & Flat metric & - \\
$k=0$ & - & Ill-defined metric & - \\
$k=1$ & - & Flat metric & $\sqrt{2 m}$ \\
\hline
\end{tabular}

Table A2. The location (given by $\Gamma r$ ) of the horizons with the positive $5 \mathrm{~d}$ cosmological constant $(\varepsilon=1)$.

\begin{tabular}{llll}
\hline & $m<0$ & $m=0$ & $m>0$ \\
\hline$k=-1$ & $\sqrt{-1+\sqrt{1-4 m \Gamma^{2}}}$ & - & - \\
$k=0$ & $\sqrt[4]{-4 m \Gamma^{2}}$ & - & - \\
$k=1$ & $\sqrt{1+\sqrt{1-4 m \Gamma^{2}}}$ & $\sqrt{2}$ & $\sqrt{1 \pm \sqrt{1-4 m \Gamma^{2}}}$ \\
\hline
\end{tabular}

Table A3. Same as in the table A2 with $\varepsilon=-1$.

\begin{tabular}{llll}
\hline & $m<0$ & $m=0$ & $m>0$ \\
\hline$k=-1$ & $\sqrt{1 \pm \sqrt{1+4 m \Gamma^{2}}}$ & $\sqrt{2}$ & $\sqrt{1+\sqrt{1+4 m \Gamma^{2}}}$ \\
$k=0$ & - & - & $\sqrt[4]{4 m \Gamma^{2}}$ \\
$k=1$ & - & - & $\sqrt{-1+\sqrt{1+4 m \Gamma^{2}}}$ \\
\hline
\end{tabular}

\section{Appendix A. Horizons in the spacetime (1)}

The metrics with constant curvature (1) admitting branes with constants spatial curvature for the various possible values of $\varepsilon$ and $k$ and sign of the mass parameter $m$ in certain cases describe black holes with horizons given in the tables A1-A3.

\section{Appendix B. The Killing algebra of the horizon metric}

The solution of the Killing equation gives the following independent Killing vectors for the horizon metric (6), written in the coordinate basis $(t, \rho, \chi, \theta, \varphi)$ :

$$
\begin{aligned}
K_{\mathbf{1}} & =(0,0,0,0,1) \\
K_{\mathbf{2}} & =(0,0,0,-\cos \varphi, \cot \theta \sin \varphi) \\
K_{\mathbf{3}} & =(0,0,0, \sin \varphi, \cot \theta \cos \varphi) \\
K_{\mathbf{4}} & =\left(0,0,-\cos \theta, \sin \theta \partial_{\chi} \ln \mathcal{H}, 0\right) \\
K_{\mathbf{5}} & =\left(0,0, \sin \theta \sin \varphi, \cos \theta \sin \varphi \partial_{\chi} \ln \mathcal{H}, \frac{\cos \varphi}{\sin \theta} \partial_{\chi} \ln \mathcal{H}\right) \\
K_{\mathbf{6}} & =\left(0,0, \sin \theta \cos \varphi, \cos \theta \cos \varphi \partial_{\chi} \ln \mathcal{H},-\frac{\sin \varphi}{\sin \theta} \partial_{\chi} \ln \mathcal{H}\right) \\
K_{\mathbf{7}} & =(1,0,0,0,0) \\
K_{\mathbf{8}} & =(t,-\rho, 0,0,0)
\end{aligned}
$$




$$
K_{9}=\left(\frac{t^{2}}{2}+\frac{1}{2 \rho^{2}},-t \rho, 0,0,0\right)
$$

The Killing vectors $K_{1-6}$ are the usual cosmological symmetries (representing rotations and quasi-translations), and they are space-like. In order to find out the causal character of the rest of the Killing vectors we calculate their length in the horizon metric

$$
\begin{aligned}
& g\left(K_{\mathbf{7}}, K_{\mathbf{7}}\right)=\frac{\varepsilon}{2 \Gamma^{2}} \rho^{2}, \\
& g\left(K_{\mathbf{8}}, K_{\mathbf{8}}\right)=\frac{\varepsilon}{2 \Gamma^{2}}\left(\rho^{2} t^{2}-1\right), \\
& g\left(K_{\mathbf{9}}, K_{\mathbf{9}}\right)=\frac{\varepsilon}{8 \Gamma^{2} \rho^{2}}\left(\rho^{2} t^{2}-1\right)^{2} .
\end{aligned}
$$

It is obvious that $K_{7}$ and $K_{9}$ are time-like for $\varepsilon=-1$ and space-like for $\varepsilon=1$, while the causal character of $K_{\mathbf{8}}$ depends on the sign of the product $\varepsilon\left(\rho^{2} t^{2}-1\right)$. The horizon metric is static in all cases excepting when $\varepsilon=1$ and $\rho^{2} t^{2}>1$. Also the locus $\rho t= \pm 1$ is a Killing horizon for $K_{\mathbf{8}}$.

The Killing vectors $K_{1-7}$ are also Killing vectors for the black hole metric (1). While $K_{1-6}$ remain space-like, the causal character of $K_{7}$ depends on the region of spacetime: calculated with the metric (1) $g\left(K_{7}, K_{7}\right)=-f$. Therefore $K_{7}$ is time-like if there is no horizon; timelike above the horizon and space-like below, if there is one horizon; and time-like above the exterior horizon and below the inner horizon, space-like between the two horizons, when there are two horizons (it is time-like everywhere excepting the horizon for degenerated horizons); finally on any horizon is null, thus the event horizons are also Killing horizons for $K_{7}$ in the black hole metrics (1), a property which is lost in the approximate horizon metric.

Having the additional $K_{\mathbf{8 , 9}}$ Killing vectors, the horizon metric has more symmetries, than the full black hole metric.

The Killing algebra is given by

$$
\begin{aligned}
& {\left[K_{\mathbf{i}}, K_{\mathbf{j}}\right]=\varepsilon_{i j k} K_{\mathbf{k}},} \\
& {\left[K_{\mathbf{3}+\mathbf{i}}, K_{\mathbf{3 + \mathbf { j }}}\right]=\varepsilon \varepsilon_{i j k} K_{\mathbf{k}},} \\
& {\left[K_{\mathbf{i}}, K_{\mathbf{3 + \mathbf { j }}}\right]=\varepsilon_{i j k} K_{\mathbf{3 + \mathbf { k }}},} \\
& {\left[K_{\mathbf{6}+\mathbf{i}}, K_{\mathbf{j}}\right]=0=\left[K_{\mathbf{6 + \mathbf { i }}}, K_{\mathbf{3 + \mathbf { j }}}\right],} \\
& {\left[K_{\mathbf{7}}, K_{\mathbf{8}}\right]=K_{\mathbf{7}},} \\
& {\left[K_{\mathbf{8}}, K_{\mathbf{9}}\right]=K_{\mathbf{9}},} \\
& {\left[K_{\mathbf{7}}, K_{\mathbf{9}}\right]=K_{\mathbf{8}},}
\end{aligned}
$$

and is classified in table B1.

The Killing vectors $K_{1-6}$ of the horizon metric and $K_{1-6}^{\mathrm{GM}}$ of the GM metric are identical and the Killing vectors $K_{7-9}$ are also related to the Killing vectors $K_{7-9}^{\mathrm{GM}}$ (specified for $A=1$ ). In order to establish these relations, $K_{\mathbf{7}-9}$ have to be transformed in the GM coordinate basis by the coordinate transformations derived in sections 3 and 4

(a) Applying the coordinate transformation (14) on $K_{7-9}$ we find

$$
K_{7}^{\mathrm{GM}}=K_{\mathbf{7}}, \quad K_{\mathbf{8}}^{\mathrm{GM}}=-K_{\mathbf{9}}, \quad K_{\mathbf{9}}^{\mathrm{GM}}=-K_{\mathbf{8}} .
$$


Table B1. Killing algebras of the black hole metric (upper row) and horizon metric (bottom row) for $\varepsilon= \pm 1$.

\begin{tabular}{lll}
\hline$\varepsilon$ & 1 & -1 \\
\hline$K_{1-7}$ & so $(4) \oplus \mathbb{R}$ & so $(1,3) \oplus \mathbb{R}$ \\
$K_{1-9}$ & so $(4) \oplus \operatorname{so}(1,2)$ & so $(1,3) \oplus \operatorname{so}(1,2)$ \\
\hline
\end{tabular}

(b1) Applying (23)

$$
\begin{aligned}
& K_{7}^{\mathrm{GM}}=K_{\mathbf{8}}, \\
& K_{\mathbf{8 , 9}}^{\mathrm{GM}}=D K_{\mathbf{9}} \pm \frac{1}{2 D} K_{7} .
\end{aligned}
$$

(b2) Applying (24)

$$
\begin{aligned}
& K_{7}^{\mathrm{GM}}=K_{\mathbf{8}}, \\
& K_{\mathbf{8 , 9}}^{\mathrm{GM}}=-D K_{\mathbf{9}} \pm \frac{1}{2 D} K_{7} .
\end{aligned}
$$

(b3) Finally applying (25)

$$
\begin{aligned}
& K_{7}^{\mathrm{GM}}=\mathrm{i} K_{\mathbf{8}}, \\
& K_{\mathbf{8}}^{\mathrm{GM}}=D K_{\mathbf{9}}-\frac{1}{2 D} K_{7}, \\
& K_{\mathbf{9}}^{\mathrm{GM}}=\mathrm{i}\left(D K_{\mathbf{9}}+\frac{1}{2 D} K_{7}\right) .
\end{aligned}
$$

Thus in the cases (a), (b1), (b2) $K_{7-9}^{\mathrm{GM}}$ are linear combinations with constant real coefficients of $K_{\mathbf{7 - 9}}$, while in the case (b3) the linear combination is complex.

\section{Appendix C. General relativistic analogy: the Bertotti-Robinson metric as the horizon region of the extremal Reissner-Nordström spacetime}

The Reissner-Nordström metric describes the spherically symmetric, static electro-vacuum exterior of a point mass $m$ with electric charge $q$. The two horizons degenerate into a single one located at $r=m$ in the extremal case, when $q=m$. Then the line element takes the form

$$
\mathrm{d} s_{\mathrm{RN}}^{2}=-\left(1-\frac{m}{r}\right)^{2} \mathrm{~d} t^{2}+\left(1-\frac{m}{r}\right)^{-2} \mathrm{~d} r^{2}+r^{2} \mathrm{~d} \Omega^{2},
$$

with $\mathrm{d} \Omega^{2}$ the infinitesimal solid angle on the unit 2-sphere. In order to approximate the metric (C.1) in the vicinity of the horizon, it is useful to introduce a new coordinate $\rho=r-m$ [30], in terms of which the line element (C.1) becomes

$$
\mathrm{d} s_{\mathrm{RN}}^{2}=-\left(\frac{\rho}{\rho+m}\right)^{2} \mathrm{~d} t^{2}+\left(\frac{\rho}{\rho+m}\right)^{-2} \mathrm{~d} \rho^{2}+(\rho+m)^{2} \mathrm{~d} \Omega^{2} .
$$

Close to the horizon $(\rho \approx 0)$ the extremal Reissner-Nordström spacetime is approximated as

$$
\mathrm{d} s_{h R N}^{2}=-\left(\frac{\rho}{m}\right)^{2} \mathrm{~d} t^{2}+\left(\frac{\rho}{m}\right)^{-2} \mathrm{~d} \rho^{2}+m^{2} \mathrm{~d} \Omega^{2} .
$$

10 
The sequence of transformations (i) $t^{\prime}=\mathrm{i} t$; (ii) $\rho=m \exp \left(-\tau^{\prime}\right) \cosh z, t^{\prime}=m \exp \left(\tau^{\prime}\right) \tanh z$; (iii) $\tau=\mathrm{i} \tau^{\prime}$ brings the metric into the form [30]

$$
\mathrm{d} s_{h \mathrm{RN}}^{2}=m^{2}\left[-\cosh ^{2} z \mathrm{~d} \tau^{2}+\mathrm{d} z^{2}+\mathrm{d} \Omega^{2}\right] .
$$

The sequence of coordinate transformations can also be given as

$$
\rho=m \exp (\mathrm{i} \tau) \cosh z, \quad t=-\mathrm{i} m \exp (-\mathrm{i} \tau) \tanh z,
$$

with the inverse

$$
z=\operatorname{arcsinh}\left(\frac{\mathrm{i} \rho t}{m^{2}}\right), \quad 2 \mathrm{i} \tau=\ln \frac{m^{2} \rho^{2}}{m^{4}-t^{2} \rho^{2}} .
$$

The energy-momentum tensor of the extremal Reissner-Nordström spacetime in the $(t, r, \theta, \varphi)$ coordinate system is

$$
T_{b}^{a}=\frac{m^{2}}{r^{4}} \operatorname{diag}(-1,-1,1,1)
$$

With the coordinate $\rho=r-m$ and for $\rho \approx 0$ this approximates as

$$
T_{b}^{a}=\frac{1}{m^{2}} \operatorname{diag}(-1,-1,1,1)
$$

The approximate horizon metric (C.3) solves the Einstein equations for the above energymomentum tensor. Moreover, after applying the complex coordinate transformation (C.5) the energy-momentum tensor will have the same form (C.8).

This describes a pure electric field, as required. In order to see this, we note that in the coordinates $(t, r, \theta, \varphi)$ the only non-vanishing components of the Maxwell tensor for the Reissner-Nordström spacetime are

$$
F_{t r}=-F_{r t}=-\frac{q}{r^{2}}
$$

In the extremal case $q=m$ and the degenerated horizon is at $r=m$, such that the nonvanishing components of the Maxwell tensor become

$$
F_{t r}=-F_{r t}=-\frac{1}{m} .
$$

The Bertotti-Robinson spacetime [28, 29] represents the product of two Riemannian 2surfaces with constant curvature radius, generated by a covariantly constant electromagnetic field in the presence of a cosmological constant. Its generic form is given by the line element

$$
\mathrm{d} s_{\mathrm{BR}}^{2}=-\left(1+\frac{x^{2}}{r_{+}^{2}}\right) \mathrm{d} t^{2}+\left(1+\frac{x^{2}}{r_{+}^{2}}\right)^{-1} \mathrm{~d} x^{2}+r_{-}^{2} \mathrm{~d} \Omega^{2} .
$$

By performing the coordinate transformations

$$
\operatorname{arcsinh} \frac{x}{r_{+}}=z, \quad t=r_{+} \tau
$$

which gives $1+x^{2} / r_{+}^{2}=\cosh ^{2} z$, the Bertotti-Robinson metric becomes

$$
\mathrm{d} s_{\mathrm{BR}}^{2}=r_{+}^{2}\left[-\cosh ^{2} z \mathrm{~d} \tau^{2}+\mathrm{d} z^{2}\right]+r_{-}^{2} \mathrm{~d} \Omega^{2},
$$

which agrees with the metric (C.4) in the case when the two Riemannian surfaces have the same curvature radii

$$
r_{+}=r_{-}=m \text {. }
$$

The equality of the two curvature radii is equivalent with the vanishing of the cosmological constant in the Bertotti-Robinson solution, which therefore represents the spacetime generated by a pure electromagnetic field. 
The Maxwell tensor for the Bertotti-Robinson spacetime is given by equation (17) in [28], representing parallel electric and magnetic fields. In the $(\tau, z, \theta, \varphi)$ coordinate system the energy-momentum tensor is given by

$$
T_{b}^{a}=\mu \operatorname{diag}(-1,-1,1,1)
$$

where $\mu=1 / \mathrm{m}^{2}$ (see (C.8)) is related to the two invariants of the electromagnetic field as

$$
\mu^{2}=\left(\mathbf{h}^{2}-\mathbf{e}^{\mathbf{2}}\right)^{2}+(2 \mathbf{e h})^{2} .
$$

Since the energy-momentum tensor only depends on $\rho$, this is what the geometry determinates. Thus another key piece of information about the electromagnetic field, represented by the parameter

$$
\alpha=-\frac{1}{2} \arctan \frac{2 \mathbf{e h}}{\mathbf{h}^{2}-\mathbf{e}^{\mathbf{2}}},
$$

remains undeterminated. With the convenient choice of $\alpha$ the electromagnetic field can be chosen as a pure electric field with $\mathbf{e}^{2}=1 / \mathrm{m}^{2}$ in perfect agreement with the source (C.10) of the horizon metric.

\section{References}

[1] Shiromizu T, Maeda K and Sasaki M 2000 Phys. Rev. D 62024012

[2] Gergely L Á 2003 Phys. Rev. D 68124011

[3] Maartens R 2004 Living Rev. Rel. 71

[4] Dadhich N, Maartens R, Papadopoulos P and Rezania V 2000 Phys. Lett. B 4871

[5] Germani C and Maartens R 2001 Phys. Rev. D 64124010

[6] Deruelle N 2001 Stars on branes: the view from the brane Preprint gr-qc/0111065

[7] Ovalle J 2007 Searching exact solutions for compact stars in braneworld: a conjecture Preprint gr-qc/0703095

[8] Bruni M, Germani C and Maartens R 2001 Phys. Rev. Lett. 87231302

[9] Dadhich N and Ghosh S G 2001 Phys. Lett. B 5181

[10] Dadhich N and Ghost S G 2002 Phys. Lett. B 538233

[11] Casadio R and Germani C 2005 Prog. Theor. Phys. 11423

[12] Pal S 2006 Phys. Rev. D 74124019

[13] Gergely L Á 2007 J. Cosmol. Astropart. Phys. JCAP07(2007)027

[14] Barrow J D and Tsagas C G 2004 Phys. Rev. D 69064007

[15] Gergely L Á 2006 Phys. Rev. D 74024002

[16] Gergely L Á and Képíró I 2007 J. Cosmol. Astropart. Phys. JCAP07(2007)007

[17] Binétruy P, Deffayet C, Ellwanger U and Langlois D 2000 Phys. Lett. B 477285

[18] Gergely L Á and Maartens R 2002 Class. Quantum Grav. 19213

[19] Eddington A S 1930 Mon. Not. R. Astron. Soc. 90668

[20] Barrow J D, Ellis G F, Maartens R and Tsagas C 2003 Class. Quantum Grav. 20 L155

[21] Iakubovskyi D and Shtanov Y 2005 Class. Quantum Grav. 222415

[22] Boehmer C G, Hollenstein L and Lobo F S N 2007 Phys. Rev. D 76084005

[23] Parisi L, Bruni M, Maartens M and Vandersloot K 2007 Preprint arXiv:0706.4431v1

[24] Bowcock P, Charmousis C and Gregory R 2000 Class. Quantum Grav. 174745

[25] Gregory R 2008 Braneworld black holes Preprint arXiv:0804.2595

[26] Gergely L Á 2004 Class. Quantum Grav. 21935

[27] Bronnikov K A and Melnikov V N 1995 Gen. Rel. Grav. 27465

[28] Bertotti B 1959 Phys. Rev. 1161331

[29] Robinson I 1959 Bull. Acad. Polon. Sci. Ser. Mat. Fis. Astr. 7351

[30] Brill D R 1992 Phys. Rev. D 461560

[31] Seahra S S and Wesson P S 2003 J. Math. Phys. 44664

[32] Mukohyama S, Shiromizu T and Maeda K 2000 Phys. Rev. D 62024028 Mukohyama S, Shiromizu T and Maeda K 2001 Phys. Rev. D 63029901 (erratum)

[33] Long J C et al 2003 Nature 421922 Gundlach J H et al 2007 Phys. Rev. Lett. 98150801 Kapner D J, Cook T S and Adelberger E G 2007 Phys. Rev. Lett. 98021101 